\title{
A multivalent vaccine candidate targeting enterotoxigenic Escherichia coli fimbriae for broadly protecting against porcine post-weaning diarrhea
}

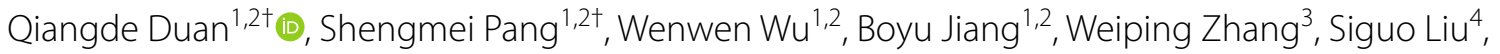
Xiaojun Wang ${ }^{4}$, Zhiming Pan ${ }^{1,2^{*}}$ and Guoqiang Zhu $u^{1,2^{*}}$

\begin{abstract}
Fimbriae-mediated initial adherence is the initial and critical step required for enterotoxigenic Escherichia coli (ETEC) infection. Therefore, vaccine candidates have been developed that target these fimbriae and induce specific antifimbriae antibodies to block initial ETEC attachment. While this vaccine effectively protects against ETEC-associated post-weaning diarrhea (PWD), developing a broadly effective vaccine against initial ETEC attachment remains a challenging problem, owing to the immunological heterogeneity among these antigens. Here, we applied multi-epitope fusion antigen (MEFA) technology to construct a FaeG-FedF-FanC-FasA-Fim41a MEFA using the adhesive subunits of predominant fimbriae $\mathrm{K} 88$ and $\mathrm{F} 18$ as the backbone, which also integrated epitopes from adhesive subunits of the rare fimbriae K99, 987P, and F41; we then generated a MEFA computational model and tested the immunogenicity of this MEFA protein in immunized mice. We next evaluated the potential of the fimbriae-targeted MEFA as a vaccine candidate to effectively prevent PWD using in vitro assessment of its anti-fimbriae, antibody-directed inhibition of bacterial adherence. Computational modeling showed that all relevant epitopes were exposed on the MEFA surface and mice subcutaneously immunized with the MEFA protein developed IgG antibodies to all five fimbriae. Moreover, anti-fimbriae antibodies induced by the MEFA protein significantly inhibited the adhesion of K88+, F18+, K99+, $987 P+$, and F41 + ETEC strains to piglet small intestinal IPEC-1 and IPEC-J2 cell lines. Taken together, these results indicate that FaeG-FedF-FanC-FasA-Fim41a MEFA protein induced specific anti-fimbriae neutralizing antibodies against the five targeted fimbriae. Critically, these results show the potential of fimbriae-targeted MEFA and indicate their promise as a broad, effective vaccine against PWD.
\end{abstract}

Keywords: ETEC, PWD, Fimbriae, MEFA, Vaccine

\section{Introduction}

Enterotoxigenic Escherichia coli (ETEC) characteristically produces two types of virulence factors: fimbriae and enterotoxins. ETEC is recognized as a major bacterial

\footnotetext{
*Correspondence: zmpan@yzu.edu.cn; yzgqzhu@yzu.edu.cn

${ }^{\dagger}$ Qiangde Duan and Shengmei Pang contributed equally to this work

${ }^{1}$ College of Veterinary Medicine (Institute of Comparative Medicine), Yangzhou University, 12 East Wenhui Road, Yangzhou 225009, China

Full list of author information is available at the end of the article
}

cause of diarrhea in young children in lower-income countries as well as in international travelers; it is also a cause of diarrhea in neonatal and post-weaning piglets [1-3]. Fimbriae and enterotoxins are the two prominent virulence determinants that contribute to ETEC-associated diarrhea. Fimbriae promote the pathogen's initial binding to specific receptors on the target host cell and subsequent colonization of the host's intestinal epithelial cells. Molecular epidemiological studies, from cases

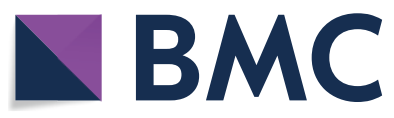

(c) The Author(s) 2020. This article is licensed under a Creative Commons Attribution 4.0 International License, which permits use, sharing, adaptation, distribution and reproduction in any medium or format, as long as you give appropriate credit to the original author(s) and the source, provide a link to the Creative Commons licence, and indicate if changes were made. The images or other third party material in this article are included in the article's Creative Commons licence, unless indicated otherwise in a credit line to the material. If material is not included in the article's Creative Commons licence and your intended use is not permitted by statutory regulation or exceeds the permitted use, you will need to obtain permission directly from the copyright holder. To view a copy of this licence, visit http://creativeco mmons.org/licenses/by/4.0/. The Creative Commons Public Domain Dedication waiver (http://creativecommons.org/publicdomain/ zero/1.0/) applies to the data made available in this article, unless otherwise stated in a credit line to the data. 
of calibacillosis in pigs, have indicated that post-weaning diarrhea (PWD) is primarily caused by ETEC strains expressing K88 and F18 fimbriae, with the K88ac variant of the K88 fimbria being the most prevalent $[4,5]$. Although K99, 987P, and F41 fimbriae are usually associated with neonatal diarrhea, they are occasionally found in PWD infections $[4,6,7]$. Despite the difference in the antigenic classification of $\mathrm{K} 88$ serological variants (e.g., $\mathrm{K} 88 \mathrm{ab}, \mathrm{ac}, \mathrm{ad}$ ), the major structural subunit-FaeG-has been recognized as the common adhesive subunit to all variants [8]. When it comes to the F18 fimbria, two antigenic variants-F18ab and F18ac-have been identified. Of these, the F18ac variant has been frequently related to PWD, while the F18ab variant has been more associated with porcine edema disease (ED) [9]. The adhesive subunit of the F18 fimbria is its minor subunit-FedF-and this subunit is highly conserved between the two antigenic variants [10]. Once colonization of the small intestinal epithelial cells occurs, ETEC bacteria secrete two classes of enterotoxins: heat-labile enterotoxin (LT) and heat-stable enterotoxin (ST). Collectively, these toxins stimulate the intestinal lining to secrete excessive fluid, thus causing diarrhea. The initial subsequent attachment between the adhesive subunit of the fimbriae and special receptors on host cells facilitates efficient delivery of enterotoxins and progression of infection pathogenesis, both of which play important roles in ETEC infections.

Newly weaned piglets are very susceptible to PWD, which remains a major disease in the swine industry and accounts for substantial global economic losses [1]. PWD causes a variety of symptoms in newly weaned piglets, including weight loss, profuse watery diarrhea, and even acute death, and remains a major challenge for the industry. Various prevention strategies have been tried to control and prevent PWD, including treatment with antibiotics [11], passive administration with specific antibodies [12], dietary supplementation [13], genetic breeding programs to generate ETEC-resistant stock [14] and vaccination [15]. However, none have been effective in protecting against PWD. Currently, treatment with antibiotics during the first 2 weeks post-weaning can relieve ETEC-associated diarrhea and other clinical symptoms. However, inappropriate and excessive use of antibiotics has led to animal health problems such as induced antimicrobial resistance in bacteria, which can cause diseases in animals [16]. Oral administration of specific anti-fimbriae antibodies to pregnant sows can provide a small amount of protection during feeding, but is both expensive and labor intensive [17]. Regarding genetic breeding strategies, it remains difficult to not only identify specific genetic markers, but also apply them to screen ETEC resistant or susceptible pigs. Given this persistent problem, a strong need for alternative strategies for the prevention and treatment of PWD remains. Vaccination is considered an ideal and effective approach to better control and protect against ETEC-caused PWD. One of ETEC's major virulent determining fimbria has received significant attention owing to its essential role in ETEC initial adherence and good immunogenicity [2]. In the past decades, vaccine development efforts have focused primarily on using ETEC fimbriae as antigens to induce production of anti-fimbriae antibodies. These antibodies then block the initial adherence of ETEC. Pigs have been vaccinated with cocktail products that contain the whole, dead ETEC bacteria expressing various fimbriae, avirulent strains expressing ETEC fimbriae, purified fimbriae, or fimbriae adhesive subunit. In all cases, these vaccines were able to resist PWD caused by ETEC expressing the corresponding fimbriae $[18,19]$. Recently, a commercialized, live, and non-pathogenic vaccine for F4+ and F18+ ETEC was shown to protect newly weaned piglets who had been challenged with both F4+ and F18+ ETEC. Impressively, this protection was achieved with only one, oral dose [20]. Vaccines administrated via the oral route will rapidly trigger mucosal immunity and induce production of antigen-specific sIgA antibodies, which will block the colonization of the pathogenic bacteria and protect against ETEC-caused PWD. However, these vaccines are unable to provide cross-protection against PWD caused by ETEC expressing different fimbriae, owing to the heterogeneity of these antigens. Though the vast majority of ETEC causing PWD mostly express K88 or F18 fimbriae, ETEC expressing K99, 987P and F41 fimbriae are usually found in ETEC caused neonatal diarrhea and also occasionally in PWD infection. Luppi et al. [6] reported that the prevalence rate of K99, 987P and F41 fimbriae among ETEC isolated from cases of PWD was $0.6 \%, 0.6 \%$ and $0.3 \%$, respectively in Europe. In China, F5 (12\%), F6 (7.2\%) and F41 (6\%) were also frequently detected, suggesting that these fimbriae were closely related to ETEC isolated from piglets with PWD [7]. Therefore, a vaccine that encompasses all five ETEC fimbriae would be more effective in providing holistic protection against ETEC-driven PWD.

In this study, we applied an epitope- and structurebased vaccinology platform to develop a fimbriae-targeted, multi-epitope fusion antigen (MEFA) protein. The MEFA protein carried all five porcine ETEC fimbriae epitopes and used both the FaeG subunit of the K88 fimbria and the FedF subunit of the F18 fimbria as its backbone. Immunogenicity of the MEFA fimbriae was tested using specific anti-fimbriae antibodies obtained from immunized mouse serum. Furthermore, the level of in vitro inhibition of ETEC adherence to piglet small intestinal epithelial cells by MEFA-induced anti-fimbriae antibodies was also determined. Finally, we also 
evaluated the potential for using these fimbriae MEFA in establishing an effective, broad-spectrum vaccine to prevent PWD.

\section{Materials and methods}

Bacterial strains, plasmids, and cell line culture conditions

The bacterial strains and plasmids used in this study are listed in Table 1. Wild-type porcine ETEC strains expressing K88, K99, 987P, F18, or F41 fimbriae (China Institute of Veterinary Drugs Control, CIVDC) were used as templates for PCR amplification of fimbriae adhesive subunit genes and also used in in vitro porcine ETEC antibody adherence inhibition assays. Expression vector pET28 $\alpha+$ (Novagen, Madison, WI) and E. coli strains DH5 $\alpha$ and BL21 (DE3) were used for gene cloning and recombinant protein expression. Porcine intestinal epithelial cell line IPEC-1 and neonatal jejunal epithelial cell line IPEC-J2 were cultured in Dulbecco minimal Eagle medium (DMEM) supplemented with $10 \%$ heat-inactivated fetal bovine serum (FBS) (Gibco) and maintained in an atmosphere of $5 \% \mathrm{CO}_{2}$ at $37^{\circ} \mathrm{C}$.

\section{Construction of FaeG-FedF-F41-FanC-FasA MEFA and adhesive subunit genes}

The FaeG subunit of the K88 fimbria and the FedF subunit of the F18 fimbria were both used as the backbone for embedding nucleotide segments coding for the neutralized epitopes of the FanC subunit of the K99 fimbria, the FasA subunit of the 987P fimbria, and the Fim41a subunit of the F41 fimbria. These subunits were all identified using a web-based B-cell epitope software, as previously described [21]. Three, less antigenic epitopes in either the FaeG or FedF subunit were substituted with nucleotide fragments coding for the most antigenic epitopes of the FasA, FanC, or Fim41a subunits. The resulting faegfim41a-fanc-fasa and fedf-fasa-fim41a-fanc chimeric genes were synthesized by Takara Biotechnology Co. Ltd. (Dalin, China) after optimizing the epitope substitution and MEFA protein structure using both a tree-dimension protein modeling program and a PyMOL molecular graphics system. These two chimeric genes were then fused to the faeg-fedf-fanc-fasa-fim 41 a chimeric gene by applying splicing overlap extension (SOE) PCR with P1 and P2 primers (Table 2). The PCR primers used to amplify the fimbriae adhesive subunit genes faeg, fanc, fasa, fedf, and fim41a are listed in Table 2. The chimeric and fimbriae subunit gene products were cloned into a restriction enzyme digested pET28 $\alpha+$ vector.

\section{Expression and purification of recombinant proteins}

Recombinant ETEC fimbriae-based MEFA and fimbriae subunit proteins were expressed and purified as previously described [21]. Briefly, a single recombinant $E$. coli colony was subcultured overnight in $2 \times Y$ T medium $(2 \times$ Yeast Extract Tryptone) containing kanamycin at a final concentration of $30 \mu \mathrm{g} / \mathrm{mL}$. Bacteria were then allowed to grow at $37{ }^{\circ} \mathrm{C}$ with vigorous shaking at $220 \mathrm{rpm}$. Then, $2 \mathrm{~mL}$ of this overnight subculture were transferred to $200 \mathrm{~mL}$ of fresh $2 \times Y \mathrm{~T}$ medium supplemented with $30 \mu \mathrm{g} /$ $\mathrm{mL}$ kanamycin. When the optical density of the bacterial

Table 1 Strains and plasmids used in this study.

\begin{tabular}{|c|c|c|}
\hline Strains/plasmids & Relevant properties & Source \\
\hline BL21 & $\mathrm{F}^{-}$ompT hsdS $\left(\mathrm{r}_{\mathrm{B}}^{-} \mathrm{m}_{\mathrm{B}}^{-}\right) \mathrm{gal} \mathrm{dcm}$ & Novagen \\
\hline C83901 & wild-type O8: H19:K88ac LT/STa/STb & CIVDC \\
\hline $2134 \mathrm{P}$ & wild-type 0157:H19 F18ac/4p-/STa/STb & Casey et al. [31] \\
\hline 1592 & Wild-type 987P+ ETEC & CIVDC \\
\hline 1593 & Wild-type K99+ ETEC, STa & CIVDC \\
\hline 1594 & Wild-type F41+ ETEC & CIVDC \\
\hline 1901 & FaeG-F41-FanC-FasA MEFA recombinant strain & This study \\
\hline 1902 & FedF-FasA-F41-FanC MEFA recombinant strain & This study \\
\hline 1903 & FaeG subunit (K88 fimbriae) recombinant strain & This study \\
\hline 1904 & FanC subunit (K99 fimbriae) recombinant strain & This study \\
\hline 1905 & FasA subunit (987P fimbriae) recombinant strain & This study \\
\hline 1906 & FedF subunit (F18 fimbriae) recombinant strain & This study \\
\hline 1907 & Fim41a subunit (F41 fimbriae) recombinant strain & This study \\
\hline \multicolumn{3}{|l|}{ Plasmid } \\
\hline pET28a+ & & Novagen \\
\hline p1908 & FaeG-F41-FanC-FasA MEFA in pET28a+ & This study \\
\hline p1909 & FedF-FasA-F41-FanC MEFA in pET28a+ & This study \\
\hline p1910 & FaeG-FedF-F41-FanC-FasA MEFA in pET28a+ & This study \\
\hline
\end{tabular}


Table 2 Primers used in this study.

\begin{tabular}{ll}
\hline Primer & Sequences $\left(\mathbf{5}^{\prime}\right.$ - $\left.^{\prime}{ }^{\prime}\right)$ \\
\hline MEFA-F1 & CGCGGATCCATGAAAAAAACCCTGATCGCTCTGGC \\
MEFA-F2 & ATCACCTACTACGGACCCGGACCTGGTCGTCTGAAATAC \\
MEFA-R1 & GAGCGTCGACTTACTGGATTTCGAA AACGATCGGAAC \\
MEFA-R2 & GTATTCAGACGACCAGGTCCGGGTCGTAGTAGGTGAT \\
FasA-F & CGCGGATCCATGAGAATGAAAAAATCCGC \\
FasA-R & CAGCGTCGACTTACGGTGTACCTGCTGAA \\
FanC-F & CGCGGTCCATGAAAAAACACTGCTAGC \\
FanC-R & CAGCGTCGACTTACATATAAGTGACTAAG \\
FedF-F & CTAGCTAGCATGCGTTTAAAATATATCTTG \\
FedF-R & CAGCGTCGACTTACTGTATCTCGAAAAC \\
FaeG-F & ATTCGGGATCCATGAAA AAGAC \\
FaeG-R & CAGCGTCGACTTAGTAATAAGT \\
Fim41a-F & CGCGGATCCATGAAAAAGACTCTGATTGC \\
Fim41a-R & AACGAGCTCTTAACTATAAATAACGGTG \\
\hline
\end{tabular}

Restriction sites, BamHI or Nhel in forward primers, and Sall or Sacl in reverse primers, were underlined.

culture reached 0.6 at $600 \mathrm{~nm}\left(\mathrm{OD}_{600}\right)$, bacterial culture was induced by $1 \mathrm{mM}$ isopropyl- $\beta$-D-1-thiogalactoside (IPTG) for an additional $4 \mathrm{~h}$. Bacteria pellets were then harvested by centrifugation at $12000 \mathrm{rpm}$ for $15 \mathrm{~min}$. Total recombinant protein was extracted with a bacterial protein extraction reagent (B-PER) (Thermo Scientific, Rochester, NY). Extracted recombinant $6 \times$ His tagged inclusion body proteins were purified from the total protein extract using protino ${ }^{\mathrm{R}} \mathrm{Ni}$-TED 2000 packed columns (MACHEREY-NAGEL, Germany) according to the manufacturer's instructions. Purified MEFA protein was examined via $12 \%$ sodium dodecyl sulfate polyacrylamide gel electrophoresis (SDS-PAGE) with Coomassie blue staining and subsequent western blot with anti-FaeG monoclonal antibody (1:1000), anti-FedF serum (1:4000), anti-FanC serum (1:4000), anti-FasA serum (1:4000), and anti-Fim41a serum (1:4000).

\section{Subcutaneous immunization of mice with fimbrial MEFA}

Animal experiments were approved by and performed at the Animal Experiment Center of Yangzhou University (Yangzhou, China). All animal experiments followed the National Institute of Health guidelines for the ethical use of animals in China.

A total of 45, 8-week-old female BALB/c mice (Laboratory Animal Center of Yangzhou University, Jiangsu, China) were divided into three groups, with 15 mice per group. In group 1 , mice were $\mathrm{SC}$ co-immunized with $50 \mu \mathrm{g}$ of the FaeG-Fim41a-FanC-FasA MEFA protein combined with $50 \mu \mathrm{g}$ FedF-FasA-Fim41a-FanC. In group 2, mice were SC immunized with only $100 \mu \mathrm{g}$ of the FaeG-FedF-FanC-FasA-Fim41a MEFA protein.
An equal volume of Freund complete adjuvant (Sigma, USA) was used as the adjuvant with the primary immunization. Each mouse received two booster injections at the same dose as the primary immunization, but with Freund incomplete adjuvant (Sigma, USA). These boosters occurred at 2-week intervals. In group 3, mice were injected only with $200 \mu \mathrm{L}$ of sterile phosphate buffer saline (PBS) and were used as the control group. All mice were euthanized 2 weeks after the second booster. Blood samples were collected from each mouse both before immunization and at the time of euthanasia.

\section{Mouse serum anti-fimbriae specific Ig $G$ antibody titration}

Serum samples from each immunized or control mouse were examined for antibodies specific to FaeG, FedF, FanC, FasA, and Fim41a fimbriae subunits. This analysis was conducted using an enzyme-linked immunosorbent assay (ELISA) as previously described [22]. Briefly, $500 \mathrm{ng}$ purified FaeG, FedF, FanC, FasA, or Fim41a recombinant protein was coated onto each well of a 96-well plate (Corning, USA) and used as the coating antigens. Noncoated spots were blocked via incubation with $10 \%$ nonfat milk at $37^{\circ} \mathrm{C}$ for $1 \mathrm{~h}$, after which the plate was washed with PBST (PBS with $0.05 \%$ Tween 20). Mouse serum samples were diluted twofolds from 1:400 to 1:25,600 and examined in the aforementioned ELISA assay. Horseradish peroxidase (HRP)-conjugated goat anti-mouse IgG (1:5000; Sigma, USA) was used as the secondary antibody. The $\mathrm{OD}_{650}$ value for each well was measured after exposure to 3, 3', 5, 5'-tetramethylbenzidine (TMB) HRP color development solution (Beyotime, China) and converted into antibody titers in $\log _{10}$ form. All experiments were conducted in triplicate.

\section{Mouse serum antibody inhibition against adherence of $\mathrm{K} 88+, \mathrm{F} 18+, \mathrm{K} 99+, 987 \mathrm{P}+$, and F41+ ETEC strains to porcine intestinal epithelial cells}

Porcine small intestinal epithelial cell lines IPEC-1 and IPEC-J2 as well as wild-type ETEC strains expressing K88, F18, K99, 987P, and F41 fimbriae were used to perform in vitro antibody adherence inhibition assessment as previously described [21]. Briefly, wild-type ETEC bacteria in the logarithmic phase were harvested and resuspended in sterile PBS. Bacterial suspensions (MOI of 5 bacteria per cell) were first co-incubated with $30 \mu \mathrm{L}$ of mouse serum from each group, and then placed on a shaker with gentle agitation for $30 \mathrm{~min}$ at room temperature. Each mouse serum/bacteria mixture (final volume of $600 \mu \mathrm{L}$ ) was added to each well of a 24-well tissue culture plate, which contained a confluent monolayer of either IPEC-1 or IPEC-J2 cells. Cells were then incubated in a $\mathrm{CO}_{2}$ incubator $\left(5 \% \mathrm{CO}_{2}\right)$ at $37{ }^{\circ} \mathrm{C}$ for $1 \mathrm{~h}$, after which they were rinsed with sterile PBS 
to remove any non-adherent bacteria. Cells were then dislodged with $0.5 \%$ Triton X-100 (Solarbio life Science, China). Dislodged cells containing the adherent ETEC bacteria were serially diluted using PBS and plated on LB agar plates. The number of bacteria colonies (CFU) on LB agar plates were counted after overnight growth in a $37^{\circ} \mathrm{C}$ incubator.

\section{Statistical analysis}

Mouse serum IgG antibody titers are presented in $\log _{10}$; in vitro mouse serum antibody adherence inhibition assay was analyzed using GraphPad Prism version 6.0 (GraphPad Software, USA). Student $t$-test was used to compare data (means \pm standard deviations) between different treatment groups. Differences were considered statistically significant at $\mathrm{p}<0.05$.

\section{Results}

Fimbriae-targeted MEFA carried the epitopes of the five fimbriae subunits of porcine ETEC

In silico predictions for each fimbriae's antigens indicate two epitopes ('NVGNGSGGANIN' and 'QLKKDDRAPSNGGYK') from the K99 fimbria major subunit FanC, two epitopes ('LAAPAENNTSQAN' and 'AGNNNTGSDTKYLV PASNDTSASG') from the 987P fimbria major subunit FasA, and two epitopes ('VMAADWTEGQ PGDII' and 'WDDLSHPNYTSADKASYLSYGSGVSAG') from the F41 fimbria major subunit Fim41a. Epitopes 'FTDYEGASVELRKPDGGTNK' and 'LPRGSELSAGSAAAA' were retained in the FaeG backbone, while epitopes 'PPNAQTYPLSSGDLK' and 'YVQPDATGSW YD' were retained in the FedF backbone. The epitopes of FanC, FasA, and Fim41a were used to substitute for three less antigenic epitopes of the FaeG or FedF backbone, and to construct either the faeg-fim41a-fanc-fasa or fedf-fasa-fim41a-fanc chimeric genes. Furthermore, the faeg-fim41a-fancfasa and fedf-fasa-fim41a-fanc genes were fused to a faeg-fedf-fanc-fasa-fim41a fimbriae MEFA gene using the SOE-PCR method (Figure 1A). The FaeG-FedFFanC-FasA-Fim41a MEFA protein was then expressed, extracted and purified.

SDS-PAGE in conjunction with Coomassie blue staining revealed a protein with a molecular mass of approximately $70 \mathrm{kDa}$, which was the expected size of the fimbriae MEFA protein (Figure 1B). The fimbriae MEFA protein was recognized by an anti-FaeG monoclonal antibody as well as anti-FedF, anti-FanC, antiFasA, and anti-Fim41a antisera (Figure 1C).
All five fimbriae epitopes were displayed on the fimbriae MEFA protein surface

Three-dimension protein modeling program and PyMOL molecular graphics system were collectively used to generate a predicted structure for the FaeG-FedF-FanCFasA-Fim41a MEFA protein based on its amino acid sequences. In total, 98 models were generated and the model with the top conformer score was selected as the final model after comparison with all 98 models. Protein modeling indicates that all inserted epitopes of K99, 987P, and F41 fimbriae were displayed on the surface of the FaeG-FedF-FanC-FasA-Fim41a MEFA protein (Figure 2).

Mice SC co-immunized with FaeG-Fim41a-FanC-FasA and FedF-FasA-Fim41a-FanC MEFA proteins developed antibody responses to each fimbria subunit

Mice co-immunized with FaeG-Fim41a-FanC-FasA and FedF-FasA-Fim41a-FanC MEFA recombinant proteins developed antibody responses to the FaeG, FedF, FanC, FasA, and Fim41a fimbrial subunits (Figure 3). Mouse serum anti-FaeG, -FedF, -FanC, -FasA, and anti-Fim41a IgG titers were $4.10 \pm 0.08,4.11 \pm 0.05,4.14 \pm 0.06$, $4.17 \pm 0.08$, and $4.23 \pm 0.11\left(\log _{10}\right)$, respectively in the co-immunized group. No anti-fimbriae subunit IgG antibody responses were detected in the control group serum.

\section{Mice SC immunized with FaeG-FedF-FanC-FasA-Fim41a MEFA protein developed antibody responses to all five fimbriae adhesive subunits}

Mice immunized with FaeG-FedF-FanC-FasA-Fim41a MEFA protein were expected to develop antibodies against all five fimbriae adhesive subunit proteins present in the fimbriae-targeted MEFA proteins (Figure 4A). Indirect ELISA using recombinant fimbriae subunit proteins as coating antigens detected anti-FaeG, -FedF, -FanC, -FasA, and anti-Fim41a IgG antibody titers at $4.55 \pm 0.14$, $4.45 \pm 0.14,4.41 \pm 0.34,4.36 \pm 0.15$, and $4.40 \pm 0.13$ from the serum samples of the immunized group. No antibody responses to these fimbriae subunit antigens were detected from the serum samples of the control mice. The anti-FaeG, -FedF, -FanC, -FasA, and anti-Fim41a IgG antibody titers in the FaeG-FedF-FanC-FasA-Fim41a MEFA protein were all significantly higher than those in the co-immunized group (Figure 4B).

Serum samples from immunized mice inhibited adherence to porcine intestinal epithelial cells of ETEC bacteria expressing the corresponding fimbriae

Serum samples from mice either immunized with FaeG-FedF-FanC-FasA-Fim41a MEFA protein or 

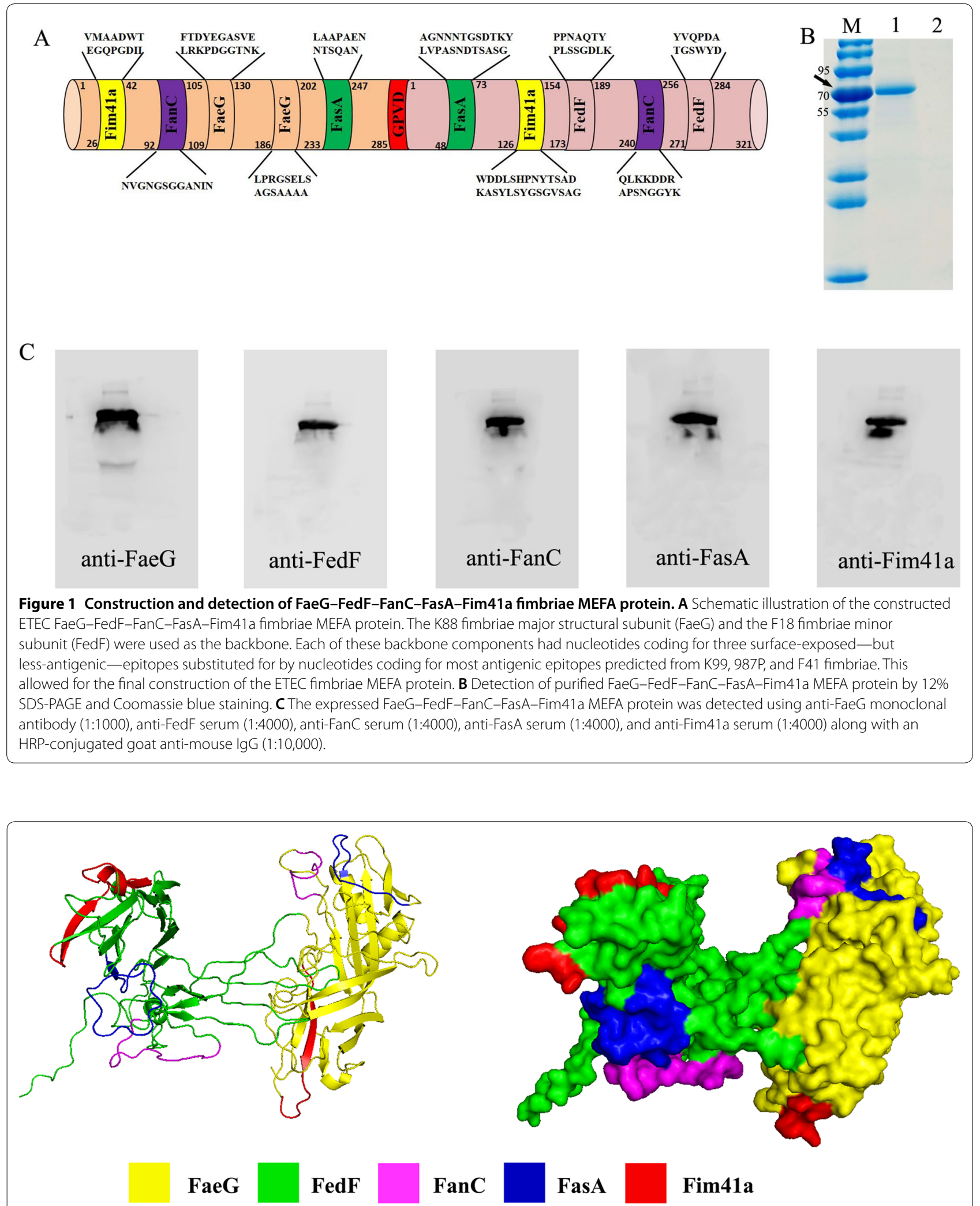

Figure 2 Computational model of FaeG-FedF-FanC-FasA-Fim41a MEFA protein. Rosetta was used to construct protein models, using the K88 fimbriae major structural subunit FaeG model (PDB ID: C2j6gA) as the template. Epitopes of the adhesive subunits of the five fimbriae are highlighted in different colors: FaeG (yellow), FedF (green), FanC (pink), FasA (blue), and Fim41a (red). 


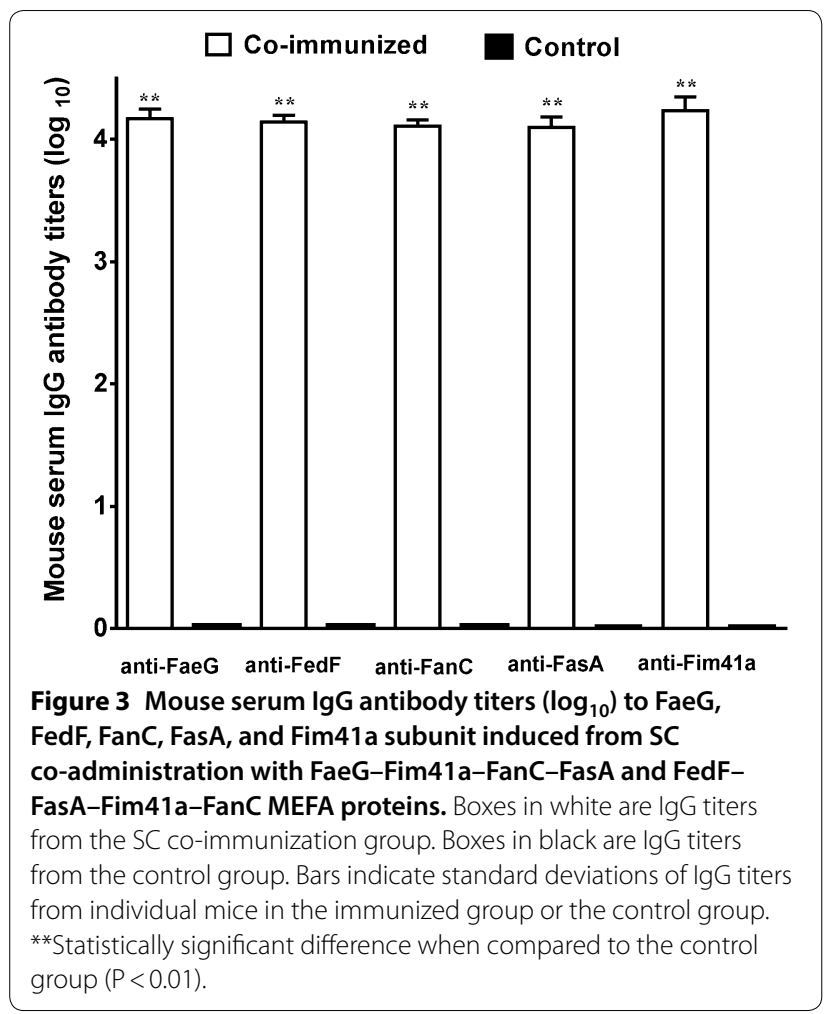

co-immunized with FaeG-Fim41a-FanC-FasA and FedF-FasA-Fim41a-FanC MEFA proteins show significantly reduced adherence of ETEC bacteria producing K88, F18, K99, 987P, or F14 fimbriae in both the IPEC-1 (Figure 5A) and IPEC-J2 cell lines (Figure 5B) $(\mathrm{P}<0.01)$, when compared to the ETEC strains incubated with the pooled serum from the control mice. Pre-incubation with serum samples obtained from the co-immunized group revealed $\mathrm{K} 88+, \mathrm{F} 18+, \mathrm{K} 99+, 987 \mathrm{P}+$, and $\mathrm{F} 14+$ ETEC strains had reduced adherence to IPEC- 1 cells by approximately $65 \%, 70 \%, 75 \%, 70 \%$, and $63 \%$, respectively; comparatively, strains had reduced adherence to IPEC-J2 cells by $57 \%, 76 \%, 84 \%, 67 \%$, and $57 \%$, respectively. These reductions were significant when compared to the same bacteria pre-incubated with serum samples from the control group. Likewise, pre-incubation with serum samples obtained from the FaeG-FedF-FanC-FasA-Fim41a immunized group revealed adherence reductions to IPEC-1 cells in the K88+, F18+, K99+, 987P+, and F14+ ETEC strains by approximately $70 \%, 71 \%, 75 \%, 72 \%$, and $65 \%$, respectively; comparatively, strains had reduced adherence to IPEC-J2 cells by $58 \%, 76 \%, 84 \%, 69 \%$, and $62 \%$, respectively. These reductions were significant when compared to the same bacteria pre-incubated with serum samples from the control group. However, serum samples from these two immunized groups had no significant differences in adherence inhibition to either IPEC-1 or IPEC-J2 cells from all five ETEC strains.
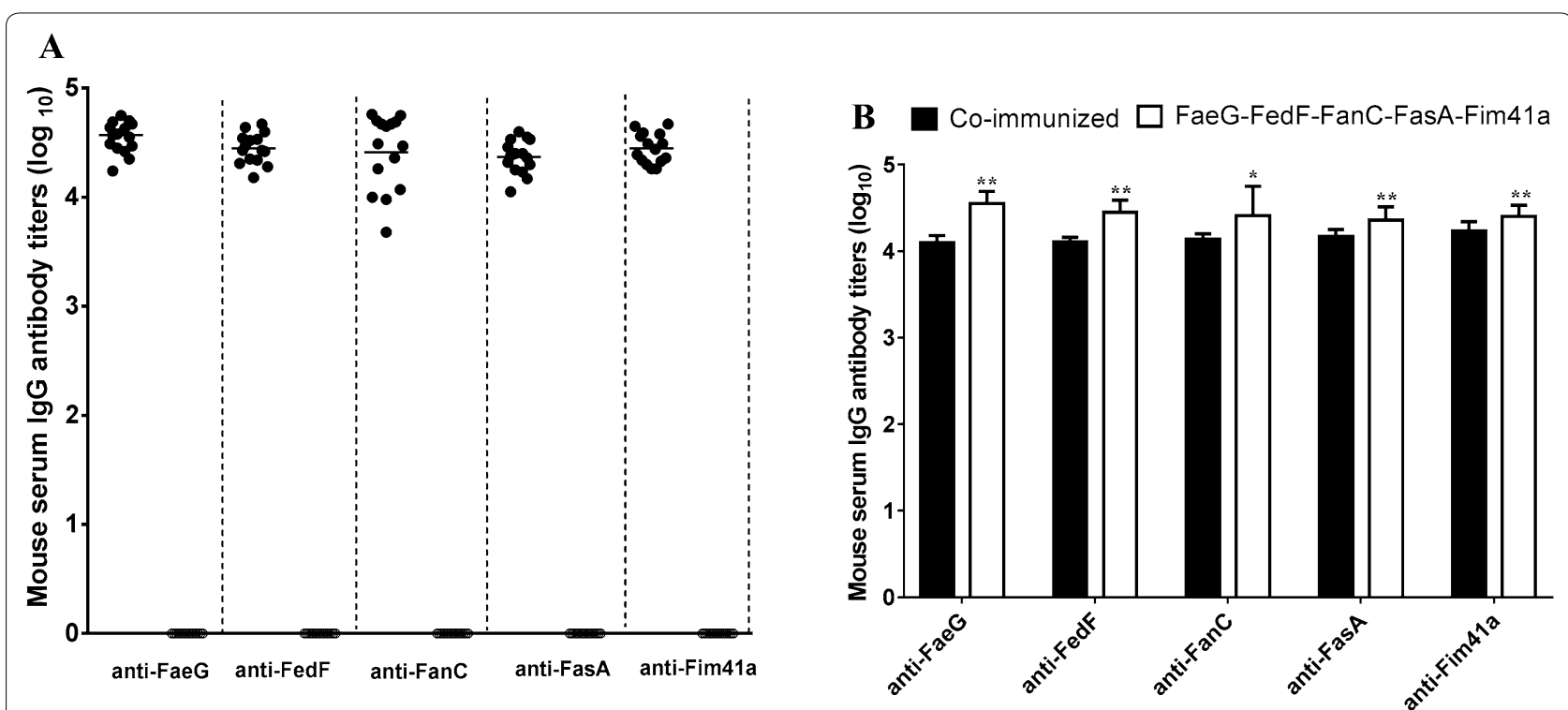

Figure 4 Mouse serum anti-fimbriae IgG antibody titers (log10) from SC immunized with FaeG-FedF-FanC-FasA-Fim41a MEFA protein. A Titration of anti-FaeG, FedF, FanC, FasA, and Fim41a lgG antibodies in serum samples of the FaeG-FedF-FanC-FasA-Fim41a MEFA protein immunized mice (filled circle) and control mice (open circle). Each circle represents the lgG titer from a single mouse. Bars indicate the mean titers of the group specific to each fimbria. Antibody titers are presented as $\log _{10}$. B Comparison of the specific anti-fimbriae antibody titers between the co-administration group and FaeG-FedF-FanC-FasA-Fim41a MEFA group. *Statistically significant difference when compared to the co-administration group $(P<0.05)$, * Statistically significant difference when compared to the co-administration group $(P<0.01)$. 

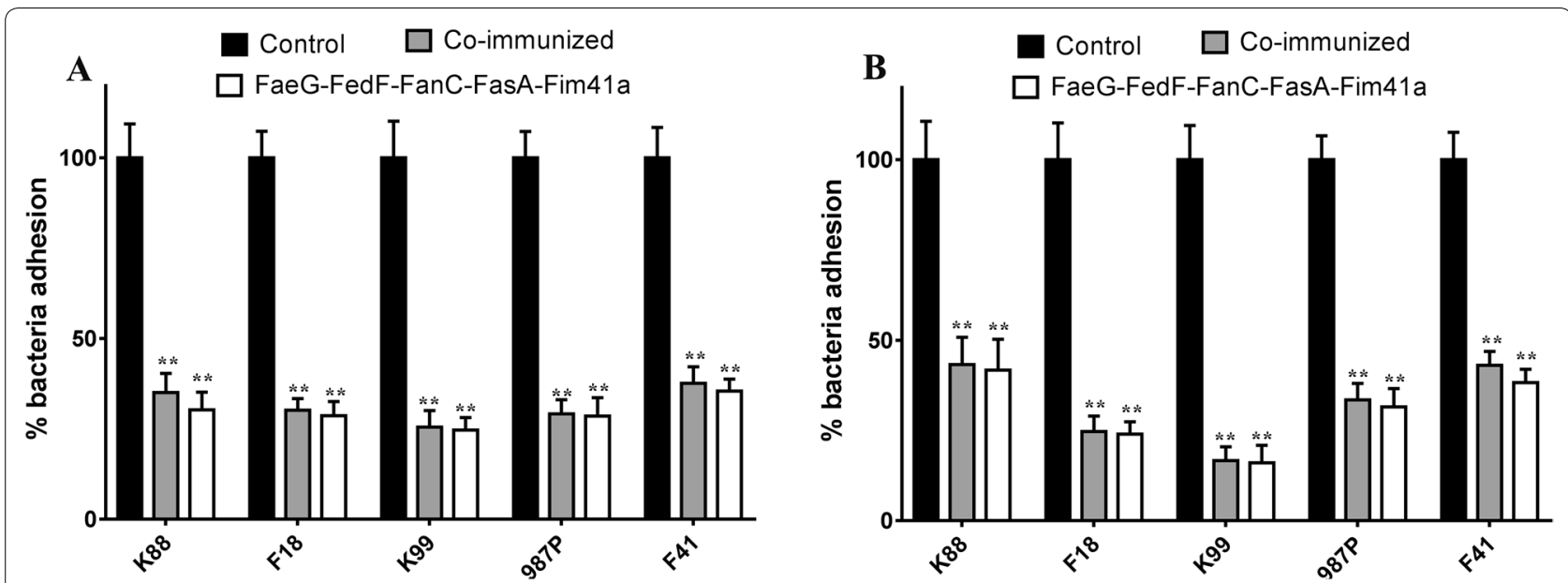

Figure 5 Anti-fimbriae antibodies from the immunized groups inhibited adherence of porcine ETEC strains to porcine small intestinal cell lines. A Serum samples from the immunized groups significantly inhibited K88+, F18+, K99+, 987P+, and F41+ ETEC strains to the IPEC-1 cell line. B Serum samples from the immunized groups significantly inhibited K88+, F18+, K99+, 987P+, and F41+ ETEC strains to the IPEC-J2 cell line. The number of adherent bacteria (CFU) was converted to percentages, with CFU from cells treated with control group serum set to $100 \%$. The results are expressed as mean \pm standard deviation of at least three independent experiments in three wells. ${ }^{* *}$ ndicates statistically significant difference when compared to the ETEC strains treated with control serum $(P<0.01)$.

\section{Discussion}

PWD caused by ETEC remains a serious problem in newly weaned piglets, resulting in considerable morbidity, mortality, and enormous global economic losses in swine husbandry [1]. In general, neonatal diarrhea in piglets can be effectively protected against by passive colostral and lactogenic immunity obtained from vaccinated sows; however, current measures have not shown effectiveness against ETEC-driven PWD in pigs [15]. To date, vaccination has been considered an effective and probably the most sustainable strategy to prevent against ETEC-associated PWD $[1,23]$. The mechanism by which the vaccine works is based on its ability to block fimbriamediated initial ETEC adhesion to the pig's enterocytes. Moreover, epidemiologic studies have shown that both K88 and F18 fimbriae are more prevalent than other fimbriae in porcine ETEC strains associated with PWD $[4,5]$. Fimbriae are also thought to be very good particle immunogens because they are proteinaceous and contain a set of epitopes that are repeated $10^{2}$ to $10^{3}$ times on each fimbrial thread [24]. Based on this concept, various active immunization studies have focused on the relevant K88 and F18 fimbriae antigens, including purified fimbriae, recombinant fimbrial adhesive subunits, or fimbriaepositive non-pathogenic E. coli to protect piglets against PWD caused by K88+ and/or F18+ ETEC infections. Recently, it has been demonstrated that two live vaccines (Coliprotec ${ }^{\circledR} \mathrm{F} 4$ and Coliprotec ${ }^{\circledR} \mathrm{F} 4 / \mathrm{F} 18$ ) developed by Elanco protected weaned piglets from PWD caused by F4+ and F18+ ETEC [20, 25]. Importantly, oral administration of a single dose of Coliprotec ${ }^{\circledR} \mathrm{F} 4$ in newly weaned piglets at least 18 days of age protected them against an F4+ ETEC challenge until 21 days post vaccination [25]. Meanwhile, oral immunization of recently weaned piglets ( $\geq 18$ days) with one dose of live, bivalent Coliprotec ${ }^{\circledR} \mathrm{F} 4 /$ F18 protected piglets who were then challenged within 7 days of vaccination with both F4+ ETEC and F18+ ETEC. Protection against F18+ ETEC lasted for at least 21-days post-vaccination [20]. Despite this promising past work, current vaccines have failed to provide crossprotection against PWD infected by the ETEC expressing K99, 987P, and F41 fimbriae. This lack of cross-protection is because there is no cross-reactivity between $\mathrm{K} 88$, $\mathrm{K} 99$, 987P, F18, and F41 fimbriae. Though the predominant fimbrial types of ETEC isolated associated with cases of PWD are K88 and F18 fimbriae, K99, 987P, and F41 fimbriae have also been detected with different rates in different regions $[4,6,7]$. Therefore, each of these fimbria types needs to be included in the vaccine to allow for the broadest level of protection.

Unfortunately, developing better vaccines against ETEC bacterial adherence and colonization based on this targeted fimbriae approach remains difficult. The major challenge to this method is the genetically and immunologically heterogeneous fimbriae that are expressed by porcine ETEC strains [26]. Those involved in PWD include five heterogeneous fimbriae virulence factors. Moreover, the immune response induced by one of the fimbria types provides protection only against ETEC strains expressing the same type of fimbria and is unable to provide cross-protection against other ETEC strains expressing a different fimbria type. 
To overcome these aforementioned challenges, an alternative approach to vaccine development that encompasses all the adhesive subunits of the five fimbria types is urgently needed. MEFA is a structurebased vaccine development technology that combines the power of computational biology with structural biology to create multivalent vaccines that incorporate the expected protective epitopes of heterogeneous virulence factors into a single protein $[27,28]$. In this study, the single fimbriae-targeted MEFA platform incorporated the expected protective epitopes from five different types of fimbriae expressed by PWD-causing ETEC strains. This MEFA protein was then created and-after immunization with it,-elicited antibodies against each fimbria included. In vitro work also shows that it effectively neutralizes ETEC attachment to intestinal epithelial cells. Importantly, this is the first report of a fimbriae-based multivalent vaccine that is capable of inducing antibodies against all five types of ETEC fimbriae associated with PWD.

Data from our murine experiments indicate that either co-administration of FaeG-Fim41a-FanC-FasA and FedF-FasA-Fim41a-FanC MEFA proteins or monoadministration with FaeG-FedF-FanC-FasA-Fim41a MEFA protein was capable of inducing a strong immune response to each of the five ETEC fimbriae. It is worth noting that the anti-fimbriae antibody titers corresponding to each fimbria in the group immunized with only the FaeG-FedF-FanC-FasA-Fim41a MEFA protein were significantly higher than those in the co-immunized group. A previous study indicated that a MEFA toxoid carrying three copies of the STa toxoid induced higher anti-STa antibody titers than those induced by the one carrying only one copy [29]. To obtain the expected higher levels of immune responses to each fimbria, we used both FaeG and FedF subunits as the backbone, each of which carried one epitope for the FanC, FasA, and Fim41a subunits. Finally, two epitopes of each fimbria subunit were carried by the FaeG-FedF-FanC-FasAFim41a MEFA protein. Therefore, the higher antibody titers in the FaeG-FedF-FanC-FasA-Fim41a MEFA group were attributable to the MEFA presenting and displaying more epitopes at the same dose. As ETEC infections are non-invasive gastrointestinal infections, protection efficacy may depend on local mucosal immunity with anti-fimbria-specific secretory IgA in the local environment of the small intestine [15]. Since the oral route is the most preferable delivery method to obtain an active intestinal mucosal immune response, delivery of the fimbria MEFA protein by a virulent host strain and subsequent induction of small intestinal mucosal immunity is an important next step to optimize future vaccine formats.
Previous studies have shown that both a CFA/I/II/IV MEFA carrying epitopes of the major subunits of the seven most important human ETEC adhesins [30] and another type of CFA adhesin tip MEFA [22] that carried the epitopes of the tip subunit of nine important human ETEC adhesins were able to induce protective antibodies against adherence of E. coli strains to Caco-2 cells expressing the corresponding adhesin. Similarly, our data indicate that antibodies derived from both immunized groups significantly inhibited adherence of porcine ETEC bacteria expressing K88, F18, K99, 987P, and F41 fimbriae to both IPEC-1 and IPEC-J2 cell lines. These cell lines are derived from either the small intestine or jejunum of neonatal piglet and used as in vitro cell models to investigate the interaction between porcine ETEC strains and porcine intestinal epithelial cells. Our results show that a MEFA protein carrying one or two epitopes of the adhesive subunit of the included fimbriae was sufficient to induce neutralizing anti-fimbriae antibodies. These results will completely change the current view that induction of protective anti-fimbriae antibodies requires either the entirety of the fimbria antigen or the adhesive subunit of the fimbria. Moreover, our results provide additional evidence that MEFA is a promising technology for the development of effective, multivalent vaccines that are broadly protective against heterogeneous pathogens and virulence factors. This work may also rejuvenate the concept of epitope vaccines, in that multiple neutralizing epitopes from heterogeneous pathogens and/or virulence factors can be integrated into a single immunogen; importantly, this approach mimics epitope native antigenicity, forming a multivalent antigen to induce broadly protective immunity.

\section{Conclusion}

Our results indicate that in mice, either SC co-administered with FaeG-Fim41a-FanC-FasA and FedFFasA-Fim41a-FanC MEFA proteins, or immunization with FaeG-FedF-FanC-FasA-Fim41a MEFA protein alone was capable of inducing production of antibodies that cross-reacted with K88, K99, 987P, F18, and F41 fimbriae. More importantly, the derived, specific antifimbriae antibodies significantly inhibited adherence of porcine ETEC bacteria expressing these five fimbriae to porcine small intestinal epithelial cells. These findings indicate that the fimbriae-targeted MEFA is a promising candidate for the production of an efficient PWD vaccine. ETEC strains expressing K88, K99, 987P, F18, and F41 fimbriae are also an important bacterial cause of neonatal diarrhea in piglets; therefore, these fimbriae MEFA are promising vaccination approaches for sows to induce lactogenic immunity. This is another means by which piglets could be protected from neonatal 
diarrhea caused by ETEC. Future work will investigate the optimized vaccine format to elicit both systemic and mucosal immune responses, coupled with pig challenge studies to verify the fimbriae-targeted MEFAinduced protective efficacy. This future work will allow a better evaluation regarding the use of ETEC vaccine candidates against both PWD and neonatal diarrhea in piglets.

\author{
Abbreviations \\ AMR: antimicrobial resistance; B-PER: bacterial protein extraction reagent; \\ CIVDC: China Institute of Veterinary Drug Control; CFUs: colony-Forming Units; \\ DMEM: Dulbecco minimal Eagle medium; ETEC: enterotoxigenic Escheri- \\ chia coli; FBS: fetal bovine serum; IPTG: isopropyl- $\beta$-D-1-thiogalactoside; LT: \\ heat-labile enterotoxin; MEFA: multi-epitope fusion antigen; PBS: phosphate \\ buffer saline; PWD: post-weaning diarrhea; SC: subcutaneously; SDS-PAGE: \\ sodium dodecyl sulfate polyacrylamide gel electrophoresis; SOE PCR: \\ splicing overlap extension PCR; ST: heat-stable enterotoxin; TMB: 3, 3' , 5 , \\ 5'-tetramethylbenzidine.
}

\section{Acknowledgements}

The authors gratefully acknowledge the funding supports.

\section{Authors' contributions}

GQ and PZ designed the experiment, DQ and PS wrote the manuscript and performed most data analysis, WW and JB performed animal experiments, ZW, $\mathrm{LS}$ and $\mathrm{WX}$ revised the manuscript. All authors read and approved the final manuscript.

\section{Funding}

This study was supported by grants from the Chinese National Science Foundation (Grant No. 31800121, 31702242) and the 63 $3^{\text {rd }}$ China Postdoctoral Science Foundation, grants from State Key Laboratory of Veterinary Biotechnology Foundation, and a project founded by the Priority Academic Program of Development Jiangsu High Education Institution, a grant from Jiangsu Science and Technology Bureau Project (BE2017342), and the Yangzhou Science and Technology Bureau International Cooperation Project (YZ2018154).

\section{Availability of data and materials}

The datasets analyzed during the current study are available from the corresponding author on reasonable request.

\section{Ethics approval and consent to participate}

Animal experiments were approved by and performed at the Animal Experiment Center of Yangzhou University (Yangzhou, China). All animal experiments followed the National Institute of Health guidelines for the ethical use of animals in China. The permit number of the Ethics committee that approved the study is YZSY20190316.

\section{Consent for publication}

All the authors approved and gave their consent for publication of the manuscript.

\section{Competing interests}

The authors declare that they have no competing interests.

\footnotetext{
Author details

${ }^{1}$ College of Veterinary Medicine (Institute of Comparative Medicine), Yangzhou University, 12 East Wenhui Road, Yangzhou 225009, China. ${ }^{2}$ Jiangsu CoInnovation Center for Prevention and Control of Important Animal Infectious Diseases and Zoonoses, Joint International Research Laboratory of Agriculture and Agri-Product Safety of Ministry of Education of China, Yangzhou, China. ${ }^{3}$ Department of Pathobiology, University of Illinois at Urbana-Champaign, Urbana, IL 61802, USA. ${ }^{4}$ Harbin Veterinary Research Institute, Chinese Academy of Agricultural Sciences, Harbin, China.
}

Received: 15 April 2020 Accepted: 11 July 2020

Published online: 23 July 2020

\section{References}

1. Fairbrother JM, Nadeau E, Gyles CL (2005) Escherichia coli in postweaning diarrhea in pigs: an update on bacterial types, pathogenesis, and prevention strategies. Anim Health Res Rev 6(1):17-39

2. Nagy B, Fekete PZ (1999) Enterotoxigenic Escherichia coli (ETEC) in farm animals. Vet Res 30(2-3):259-284

3. Platts-Mills JA, Babji S, Bodhidatta L, Gratz J, Haque R, Havt A, McCormick BJ, McGrath M, Olortegui MP, Samie A et al (2015) Pathogen-specific burdens of community diarrhoea in developing countries: a multisite birth cohort study (MAL-ED). Lancet Glob Health 3(9):e564-e575

4. Zhang W, Zhao M, Ruesch L, Omot A, Francis D (2007) Prevalence of virulence genes in Escherichia coli strains recently isolated from young pigs with diarrhea in the US. Vet Microbiol 123(1-3):145-152

5. Frydendahl K (2002) Prevalence of serogroups and virulence genes in Escherichia coli associated with postweaning diarrhoea and edema disease in pigs and a comparison of diagnostic approaches. Vet Microbiol 85(2):169-182

6. Luppi A, Gibellini M, Gin T, Vangroenweghe F, Vandenbroucke V, Bauerfeind R, Bonilauri P, Labarque G, Hidalgo A (2016) Prevalence of virulence factors in enterotoxigenic Escherichia coli isolated from pigs with postweaning diarrhoea in Europe. Porcine Health Manage 2:20

7. Li S, Wang L, Zhou Y, Miao Z (2020) Prevalence and characterization of virulence genes in Escherichia coli isolated from piglets suffering postweaning diarrhoea in Shandong Province, China. Vet Med Sci 6(1):69-75

8. Guinee PA, Jansen WH (1979) Behavior of Escherichia coli Kantigens K88ab, K88ac, and K88ad in immunoelectrophoresis, double diffusion, and hemagglutination. Infect Immun 23(3):700-705

9. Rippinger P, Bertschinger HU, Imberechts H, Nagy B, Sorg I, Stamm M, Wild P, Wittig W (1995) Designations F18ab and F18ac for the related fimbrial types F107, 2134P and 8813 of Escherichia coli isolated from porcine postweaning diarrhoea and from oedema disease. Vet Microbiol 45(4):281-295

10. Tiels P, Verdonck F, Smet A, Goddeeris B, Cox E (2005) The F18 fimbrial adhesin FedF is highly conserved among F18 + Escherichia coli isolates. Vet Microbiol 110(3-4):277-283

11. Amezcua R, Friendship RM, Dewey CE, Gyles C, Fairbrother JM (2002) Presentation of postweaning Escherichia coli diarrhea in southern Ontario, prevalence of hemolytic E. coli serogroups involved, and their antimicrobial resistance patterns. Can J Vet Res 66(2):73-78

12. Marquardt RR, Jin LZ, Kim JW, Fang L, Frohlich AA, Baidoo SK (1999) Passive protective effect of egg-yolk antibodies against enterotoxigenic Escherichia coli K88+ infection in neonatal and early-weaned piglets. FEMS Immunol Med Microbiol 23(4):283-288

13. Amezcua MD, Friendship R, Dewey C, Weese S, de Lange CF (2007) The effect of feeding fermented liquid whey plus dextrose inoculated with specific lactic acid bacteria of pig origin to weanling pigs challenged with Escherichia coli O149:K91:F4. Vet Ther 8(3):209-222

14. Rutter JM, Burrows MR, Sellwood R, Gibbons RA (1975) A genetic basis for resistance to enteric disease caused by E. coli. Nature 257(5522):135-136

15. Melkebeek V, Goddeeris BM, Cox E (2013) ETEC vaccination in pigs. Vet Immunol Immunopathol 152(1-2):37-42

16. Smith MG, Jordan D, Chapman TA, Chin JJ, Barton MD, Do TN, Fahy VA, Fairbrother JM, Trott DJ (2010) Antimicrobial resistance and virulence gene profiles in multi-drug resistant enterotoxigenic Escherichia coli isolated from pigs with post-weaning diarrhoea. Vet Microbiol 145(3-4):299-307

17. Moon HW, Bunn TO (1993) Vaccines for preventing enterotoxigenic Escherichia coli infections in farm animals. Vaccine 11(2):213-220

18. Morgan RL, Isaacson RE, Moon HW, Brinton CC, To CC (1978) Immunization of suckling pigs against enterotoxigenic Escherichia coli-induced diarrheal disease by vaccinating dams with purified 987 or K99 pili: protection correlates with pilus homology of vaccine and challenge. Infect Immun 22(3):771-777

19. Lin J, Mateo KS, Zhao M, Erickson AK, Garcia N, He D, Moxley RA, Francis DH (2013) Protection of piglets against enteric colibacillosis by intranasal 
immunization with K88ac (F4ac) fimbriae and heat labile enterotoxin of Escherichia coli. Vet Microbiol 162(2-4):731-739

20. Nadeau E, Fairbrother JM, Zentek J, Belanger L, Tremblay D, Tremblay CL, Rohe I, Vahjen W, Brunelle M, Hellmann Ket al (2017) Efficacy of a single oral dose of a live bivalent $E$. coli vaccine against post-weaning diarrhea due to $\mathrm{F} 4$ and $\mathrm{F} 18$-positive enterotoxigenic E. coli. Vet J 226:32-39

21. Duan Q, Lee KH, Nandre RM, Garcia C, Chen J, Zhang W (2017) MEFA (multiepitope fusion antigen)-novel technology for structural vaccinology, proof from computational and empirical immunogenicity characterization of an enterotoxigenic Escherichia coli (ETEC) Adhesin MEFA. J Vaccines Vaccin 8(4):367

22. Nandre RM, Ruan X, Duan Q, Sack DA, Zhang W (2016) Antibodies derived from an enterotoxigenic Escherichia coli (ETEC) adhesin tip MEFA (multiepitope fusion antigen) against adherence of nine ETEC adhesins: CFA/l, CS1, CS2, CS3, CS4, CS5, CS6, CS21 and EtpA. Vaccine 34(31):3620-3625

23. Zhang W (2014) Progress and challenges in vaccine development against enterotoxigenic Escherichia coli (ETEC)-associated porcine post-weaning diarrhea (PWD). J Vet Med Res 1(e1006):1-13

24. Dubreuil JD, Isaacson RE, Schifferli DM (2016) Animal enterotoxigenic Escherichia coli. EcoSal Plus 7(1):1-47

25. Fairbrother JM, Nadeau E, Belanger L, Tremblay CL, Tremblay D, Brunelle M, Wolf R, Hellmann K, Hidalgo A (2017) Immunogenicity and protective efficacy of a single-dose live non-pathogenic Escherichia coli oral vaccine against F4-positive enterotoxigenic Escherichia coli challenge in pigs. Vaccine 35(2):353-360
26. Zhang W, Sack DA (2015) Current progress in developing subunit vaccines against enterotoxigenic Escherichia coli-associated diarrhea. Clin Vaccine Immunol 22(9):983-991

27. Dormitzer PR, Grandi G, Rappuoli R (2012) Structural vaccinology starts to deliver. Nat Rev Microbiol 10(12):807-813

28. Liljeroos L, Malito E, Ferlenghi I, Bottomley MJ (2015) Structural and computational biology in the design of immunogenic vaccine antigens. $J$ Immunol Res 2015:156241

29. Rausch D, Ruan X, Nandre R, Duan Q, Hashish E, Casey TA, Zhang W (2017) Antibodies derived from a toxoid MEFA (multiepitope fusion antigen) show neutralizing activities against heat-labile toxin (LT), heat-stable toxins (STa, STb), and Shiga toxin 2e (Stx2e) of porcine enterotoxigenic Escherichia coli (ETEC). Vet Microbiol 202:79-89

30. Ruan X, Knudsen DE, Wollenberg KM, Sack DA, Zhang W (2014) Multiepitope fusion antigen induces broadly protective antibodies that prevent adherence of Escherichia coli strains expressing colonization factor antigen I (CFA/I), CFA/II, and CFA/IV. Clin Vaccine Immunol 21 (2):243-249

31. Casey TA, Nagy B, Moon HW (1992) Pathogenicity of porcine enterotoxigenic Escherichia coli that do not express K88, K99, F41, or 987P adhesins. Am J Vet Res 53(9):1488-1492

\section{Publisher's Note}

Springer Nature remains neutral with regard to jurisdictional claims in published maps and institutional affiliations.
Ready to submit your research? Choose BMC and benefit from:

- fast, convenient online submission

- thorough peer review by experienced researchers in your field

- rapid publication on acceptance

- support for research data, including large and complex data types

- gold Open Access which fosters wider collaboration and increased citations

- maximum visibility for your research: over $100 \mathrm{M}$ website views per year

At BMC, research is always in progress.

Learn more biomedcentral.com/submissions 